\title{
Teaching Interpersonal Communication in the UAE
}

\author{
Lyall Crawford
}

Zayed University, UAE

The purpose of this essay is to provide a personal narrative about COM 207, Interpersonal Communication, offered in the College of Communication and Media Sciences at Zayed University. It is taken and amended from my ZU evaluation portfolio and it consists of my responses to questions that direct a self-evaluation of teaching effectiveness in this particular course. These remarks are intended to be helpful for instructors who are responsible for courses of this nature in the Gulf region, in addition to giving some pedagogical and cultural comments about teaching in this part of the world.

\section{COM 207 - Brief Narrative about the Course}

\section{Course Objectives}

COM 207 is an inaugural look at the subtleties and complexities of human interaction. The focus is on face-to-face communication, although some instances of mediated interaction are considered as well, such as certain interpersonal implications of e-mail. The overall purpose of this course is to encourage a personal examination of the communication practices that facilitate and impede the initiation, continuation, transformation, and termination of human relationships. COM 207 is based on three closely related ideas: (1) that relationships emerge from our interactions with one another, (2) that the quality of these relationships depends on how well we communicate interpersonally, and (3) that the skillfulness of our communicating and the quality of our relationships shapes the quality of our lives. In other words, effective communication within the context of our interpersonal relationships (i.e., achieving personal goals while appropriately maintaining our connections with others) may facilitate more satisfied and contented lives - this is the foundational goal of COM 207.

Specific goals or course objectives for COM 207 include, but are not limited to: (1) gaining a particular sense of what interpersonal communication means, what it entails, and why it is an important area of study; (2) achieving a better understanding of the relationship implications of our verbal and nonverbal interactions with one another, especially when it comes to the expression of feelings; (3) acquiring an appreciation for the crucial place of empathic listening and responding in our personal relationships; (4) learning constructive ways to appropriately manage stress and control anger, particularly with those closest to us; (5) becoming familiar with the importance of perspective-taking and perception-checking while interacting with others; (6) developing the ability to "frame" what happens to us, particularly during our transactions with others, so our thoughts more productively influence our experience; (7) appreciating more fully some of the cultural dimensions of interpersonal communication and relationships; and (8) putting into practice self-monitoring skills to observe and refine our communication behaviors (McCornack, 2007).

\section{Class Participants}

The students at Zayed University are female UAE nationals. The majority of students in COM 207 are typically communication majors in their first or second semester of the program, although it is not 
unusual for more senior communication students and students from other majors to enroll as well. Majors signup for this course because it may be taken as an elective within the College to partially fulfill a "leadership" requirement.

As far as I know, interpersonal communication is quite likely a new area of study for virtually all the students who enroll, so it seems safe to say that most of them have probably given very little formal or academic attention to the issues that get addressed during the course of the semester. However, this is not to say that they haven't thought about and experienced many of the interpersonal dynamics that ground this course and, to a considerable extent, what they bring to the class from their Arabic culture matches exceeding well the range of Western-based ideas and principles that tend to shape and constitute what we study. The general sociality of human beings appears to be quite global in nature so it is not surprising, I suppose, that interpersonal communication issues resonate here in a manner related to the way they play out in many Western contexts.

\section{Teaching and assessment}

COM 207 was approached more-or-less traditionally; that is, a portion of our class time was devoted to short lectures and discussions about interpersonal communication issues and, instead of major exams, I used quizzes usually administered after we completed our discussion of a chapter in the text. I typically announced there would be a quiz one week before the quiz day. Quizzes were taken during regular class time and consisted of a variety of question formats, although the majority of questions were shortanswer. The final quiz took place during the final exam time at the end of the semester.

In-class activities, worksheet assignments, and textbook exercises geared toward interpersonal communication were also used to move us towards the objectives of this course. I made a special effort to facilitate classroom discussions. The website http://www.tedtalks.com was especially useful in this regard by providing short, twenty-minute, video "talking points" by prominent people addressing topics related to our interests. These web-based mini-lectures proved to be both entertaining and informative and this website has become a valued resource for all my courses. Generally speaking, young Emirati women enrolled in COM 207 have very little difficulty relating what we consider in this course to the range of interpersonal episodes they experience on a regular basis. Consequently, in-class discussions often became quite participatory, animated, and even "useful," perhaps, in terms of the insights provided for when they left the classroom and interacted with family and friends.

\section{Achievement}

COM 207 Interpersonal Communication is sometimes referred to as a "soft skills" course, and "soft skills" can be difficult to assess. Quiz performances only suggest the interpersonal effectiveness of students enrolled in the course. Even the experiential exercises of COM 207 merely scratch the surface in terms of assessing interpersonal ability. Self-assessment and observation in uncontrived circumstances may be more sensible ways to intuit how much the course brought about productive change in the communication practices of participants.

In light of this, it's hard to say how well the students did in accomplishing the objectives of the course other than to point out that there was the usual spread in quiz scores, that some students participated more actively than others, that some self-reports seemed quite insightful, and that almost everyone in the class appeared interested in course content and easily drew parallels between in-class discussions and activities and out-of-class encounters. If the main objective of COM 207 is to improve the day-today quality of individual lives by increasing the effectiveness and satisfaction of interpersonal communication, then the most realistic assessment of course efficacy is probably best seen after the course is over, not while it is going on. 


\section{Commentary}

It's probably safe to say there's a link between teaching effectiveness and student behavior. If this is the case, then I can learn about how well I'm doing as a teacher by reflecting on the behavior of students who enroll in my classes. For the most part, I think I improve little-by-little. I find my way in small increments. And occasionally, I just muddle along. Enthusiasm and motivation wane from time to time as I move toward the end of my teaching career.

But my career has been long because I believe that teaching and learning are necessary and important cultural endeavors. My career has been long because there have been signs that others concur with this view especially, perhaps, when it comes to the study of interpersonal communication. One such sign, anecdotal and particular in nature, is this passage in a paper by B. Odjargal, a young Mongolian women, submitted to me while I was teaching in China:

"When I think about communication, I hear my father saying: 'Everything is based on communication. You can have all of the business and professional training in the world, but if you can't develop communication skills you won't get anywhere and you won't have anything worth living for."

She concludes:

"Learning about communication and developing your skills as a communicator are foundations for an effective life."

I first stepped into the classroom as a teacher in the fall of 1963. I was young and inexperienced. I learned during that first year of teaching that I needed to bring much more to the classroom than just the content of the course. It was also important to have more experience in the world so I could be more than just a source of information about an academic subject. So, in the spring of 1963, I resigned my first teaching position and became a member of the United States Peace Corps. Now, more than 40 years later and with many experiences in addition to the Peace Corps, I like to believe I have a deeper sense of myself and that more than textbook information goes into the classroom with me.

I say this because it is at the heart of how I see teaching and learning. In my opinion, a principal task of the educator is the inspiration of students. The goal, beyond providing academic skills, is to nurture an enthusiasm for the issues at hand and for learning in general - especially the kind that elevates the mind while contributing to more happy, healthy, and prosperous lives.

\section{Reference}

McCornack, Steven. (2007). Reflect \& Relate: An Introduction to Interpersonal Communication. Boston and New York: Bedford/St. Martin's. 